\title{
La integración de alumnos con necesidades educativas especiales
}

\author{
Sandra Ivonne Nieto Martínez \\ Pedro Antonio Pérez Pérez \\ Profesores investigadores \\ Universidad Autónoma de Ciudad Juárez
}

\section{Resumen}

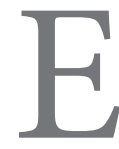

1 presente trabajo analiza las experiencias de un grupo de docentes que atienden aulas regulares, ante el proceso de integración de alumnos con Necesidades Educativas Especiales. El estudio se desarrolla a partir de la observación no participante y las entrevistas interactivas no estructuradas a los docentes.

Palabras Clave: necesidades educativas especiales, integración educativa, propuesta curricular adaptada, educación especial

\section{Introducción}

La atención de alumnos con Necesidades Educativas Especiales (NEE), es una tarea que de manera reciente se incorporó en las aulas de las escuelas regulares de educación básica, lo que representa un desafío para los maestros de grupo, ya que implica, entre otras cosas, eliminar las barreras físicas y los prejuicios que alimentan la exclusión y la discriminación, que niegan la posibilidad a estos niños y jóvenes de integrarse a los procesos de aprendizaje y de participar en la vida escolar y social. Por lo anterior, es necesario distinguir los principales rasgos del proceso de integración educativa, y las implicaciones que tienen en las situaciones psicopedagógicas que enfrentan los docentes en los contextos escolares.

Con este proyecto de investigación se pretende conocer y analizar las situaciones por las que atraviesa un grupo de docentes en la integración de alumnos con NEE; comprender su experiencia laboral e interpretar el 
significado de ésta, además de proporcionar información útil a quienes desempeñan su labor en y para las aulas regulares.

\section{El problema}

La integración educativa en aulas regulares de alumnos con alguna discapacidad, se remonta a los años sesenta (Marchesi y Martin, 1995), en el caso de México, a fines de 1970, se creó la Dirección General de Educación Especial con la finalidad de regular el sistema federal de educación especial y la formación de maestros especialistas; atendiendo a personas con discapacidades mentales y/o físicas en modalidades que funcionaban en espacios específicos separados de la educación regular.

Para 1993, como consecuencia de la suscripción del Acuerdo Nacional para la Modernización de la Educación Básica, la reforma al artículo $3^{\circ}$ constitucional y la promulgación de la Ley General de Educación, "se impulsó un importante proceso de reorientación y reorganización de los servicios de educación especial” (SEP, 2002: 12).

Dicho proceso ha permitido que la atención de los alumnos que presentan NEE deje de estar asociada exclusivamente con los servicios de educación especial; la promoción de la integración educativa, dirigió esta responsabilidad hacia la educación básica regular (SEP, 2006).

A partir de este marco, las escuelas ordinarias han comenzado a recibir alumnos con NEE, algunas de ellas apoyadas con personal especializado, por lo que actualmente la profesionalización docente requiere el desarrollo de nuevas competencias para responder ante las nuevas necesidades educativas que se gestan en las aulas regulares. Este proceso constituye un desafío para los docentes, para las instituciones educativas y para todo el Sistema Educativo Nacional.

A partir de estas consideraciones se plantea la siguiente pregunta de investigación: ¿qué situaciones enfrentan los docentes de una escuela primaria federalizada, ubicada en Cd. Juárez, Chihuahua, en la integración de alumnos con Necesidades Educativas Especiales?

\section{Estrategia metodológica}

El presente trabajo tiene una orientación metodológica de corte cualitativo, porque asegura la relación dialéctica entre el investigador y el objeto de conocimiento, es decir, el estudio es conducido por la situación, el trabajo de campo y los sujetos, prestando atención al significado de los sucesos y las acciones que se generan en el entorno educativo. Se circunscribe a un contexto escolar y su desarrollo se dirige a comprender la problemática identificada, poniendo énfasis en describir e interpretar las dinámicas que se observan a lo largo de un período prolongado, sin una intervención participativa por parte de los investigadores, con el fin de no modificar el ambiente natural.

Pourtois y Huguette exponen que "la realidad parte de la construcción de las percepciones del mundo de los agentes" (1992: 60), es decir, en este planteamiento la materia prima es valorar la experiencia docente desde el propio contexto escolar y desde la propia perspectiva de los involucrados, para no perder la esencia de cada una de las situaciones que se circunscriben en ella.

El estudio asegura la relación dialéctica

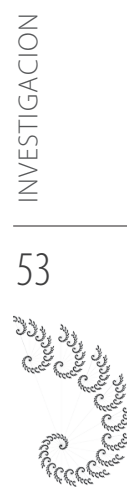


entre el investigador y el objeto de conocimiento, es decir, se mantiene un diálogo constante para describir, conocer, comprender e interpretar los fenómenos y sus significados. Hernández (1998) considera que este diálogo influye en la conceptualización o categorización que se haga del objeto de conocimiento.

Se busca la comprensión, los motivos y creencias que están detrás de la situación, de los sucesos y de las acciones de los participantes que se generan en la cotidianidad de su entorno social.

Los sujetos participantes fueron seleccionados a través de un sondeo, el cual, se dirigió a todo el conjunto de docentes frente a grupo de la escuela, donde cada uno tuvo las mismas posibilidades de formar parte de la investigación; con base a la antigüedad en el servicio educativo y en la institución, la disponibilidad para participar en la investigación y la población estudiantil con NEE, finalmente quedó confirmada por tres docentes.

El sondeo se realizó a través de un cuestionario autodirigido, donde se pretendía obtener, mediante preguntas cerradas, información sobre la disponibilidad para cooperar en la realización de la investigación.

El registro de la observación se llevó a cabo mediante una bitácora, donde se sigue un orden cronológico de todos los sucesos tal y como ocurren, para no perder la naturalidad de los hechos.

Hernández, Fernández y Baptista (2006) consideran que cada investigador tiene la facilidad de seguir su propio sistema para llenar la bitácora. Por lo tanto, las nota registradas se escribieron en un formato tamaño carta, que incluye la fecha, los sujetos, el escenario y las claves con las que se identificó a los participantes, se agregó un margen de seis centímetros del lado derecho, con el fin de realizar comentarios, preguntas e identificar aspectos relevantes de las situaciones que pueden ser típicas, semejantes, contrastantes, extrañas o inverosímiles.

Además se utilizó la entrevista interactiva no estructurada, la cual representa una fuente para recabar información en forma verbal, a través de una conversación con los distintos informantes del contexto interno y externo al aula. Mayan (2001) expone que se debe plantear una pregunta para involucrar a los participantes en el tema de investigación y el entrevistador debe mantenerla, sin embargo, se optó por utilizar una guía que sirvió para focalizar la entrevista y evitar perder el objetivo de la misma.

La investigación se desarrolló durante el periodo comprendido entre los meses de septiembre a noviembre del 2010.

El análisis e interpretación de los datos, se realizó de acuerdo con los cinco pasos que Mayan (2001) propone, los cuales son:

Codificar: es la revisión inicial de la información, donde se logra identificar y organizar extractos representativos del registro.

Categorizar: clasificar o agrupar, integrar o reintegrar las partes en relación con el todo, de manera inductiva porque son producto del registro de la descripción de la situación.

Integrar las categorías e integrar temas: establecer una relación entre las categorías y encontrar unidades de análisis.

Integrar todos los datos: reunir otros da- 
tos obtenidos cualitativamente, por ejemplo la información de las entrevistas interactivas no estructuradas.

Corolario sobre el análisis de contenido: realizar la triangulación de la información para validar la información a partir del establecimiento de argumentos significativos del objeto de conocimiento.

\section{Resultados}

Las tres unidades de análisis que representan los resultados obtenidos se muestran en la figura 1, donde además se establecen las relaciones con la información que permite plantear el objeto de estudio de una manera simbólica.

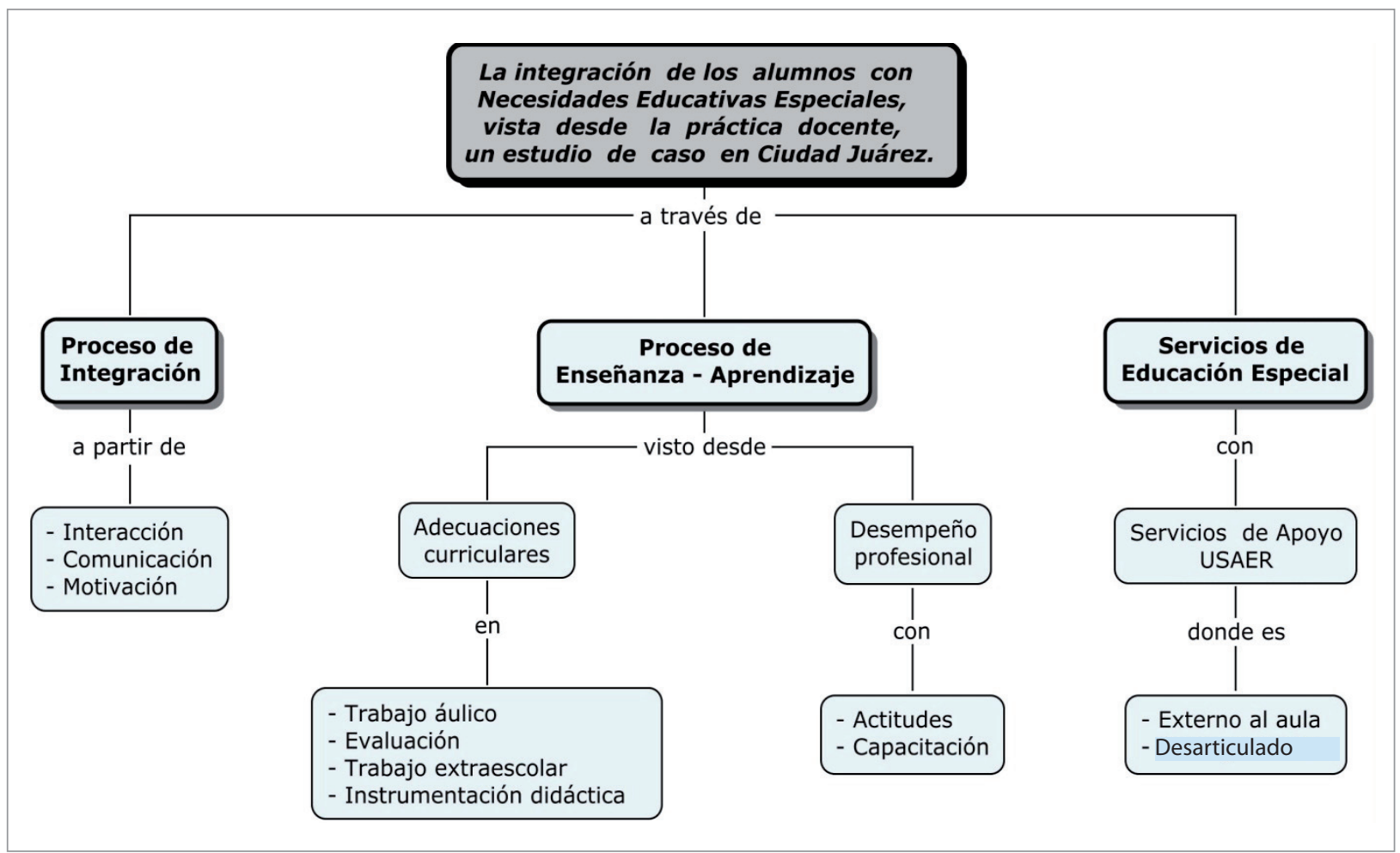

Figura 1. Unidades de análisis y subcategorías del las situaciones psicopedagógicas.

Tabla 1

Con el fin de garantizar la confidenClaves de identificación de los participantes cialidad y el anonimato, los resultados se presentan a partir de códigos que se asignaron para identificar a cada docente y al alumno $u$ alumna con NEE (ver tabla 1).

\begin{tabular}{|l|l|l|l|}
\hline Maestro (a) del aula & MA & MB & MC \\
\hline Alumnos (as) con NEE & MT & P y R & $\mathrm{J}$ \\
\hline Maestra de USAER & \multicolumn{3}{|c|}{ MU } \\
\hline Madre de Familia & \multicolumn{3}{|c|}{ MF } \\
\hline
\end{tabular}




\section{A. Proceso de integración}

En esta unidad de análisis se encuentra relacionada la integración educativa con los procesos de interacción, comunicación y motivación entre los participantes.

En la interacción se contemplan las diferentes formas en las que los alumnos con NEE y los docentes participan en el establecimiento del enlace pedagógico, que se da en función de las relaciones y los contactos recíprocos entre el maestro-alumno con NEE, alumno-alumno con NEE y alumno con NEE-grupo. La comunicación se consideró como una de las fuentes para establecer un vínculo donde se intercambien ideas, comentarios, conocimientos, pensamientos, dudas, etc., con el fin expresarse de manera verbal y no verbal.

\section{Interacción maestro- alumno con NEE}

En los tres casos se presenta una relación de dependencia del alumno con NEE hacia el docente, ya que constantemente están solicitando la aprobación del trabajo u explicación del mismo. Así mismo, se encontró que en los tres casos estudiados los docentes realizan una supervisión general sobre el trabajo de estudiantes, desplazándose por el aula, por lo que los alumnos cuando solicitan la ayuda tienden a buscarlos fuera de su lugar.

Pero también, en el caso del $\mathrm{MB}$ con el alumno $\mathrm{P}$, éste no realiza ningún esfuerzo por presentarle el trabajo al docente, por lo que permanece en su lugar esperando que vayan a revisarle o a prestarle alguna orientación sobre el trabajo que ha de realizar, lo anterior muestra que el proceso de interacción entre $\mathrm{MB}$ y $\mathrm{P}$ se realiza de forma unidireccional, puesto que la acción del alumno está supedi- tada a la instrucción del profesor. Es evidente que el hablar, para el profesor, y el escuchar, para el alumno, no expresan adecuadamente la riqueza y la complejidad de la relación pedagógica. El alumno aprende escuchando, pero también aprende leyendo, elaborando esquemas que organicen los contenidos recibidos, realizando ejercicios, reaccionando a la respuesta del profesor, discutiendo con los otros alumnos o con el profesor, etc. En resumen, el alumno aprende cuando reorganiza por sí mismo la información que recibe (Saint-Onge, 2000). Esto se complica cuando el alumno presenta NEE, pero es con ellos donde estas acciones se deben privilegiar aún más para garantizar que está aprendiendo.

\section{Interacción alumno-alumno con NEE}

En esta categoría se logró identificar que MB y MC realizan actividades encaminadas a situar la actividad constructiva del alumno con NEE con el apoyo de intervenciones verbales que realiza algún compañero del aula, el cual le brinda la ayuda para lograr avances que no tendría por sí mismo.

Lo anterior Brunner (1984) lo describe como el andamiaje, que representa la necesidad de propiciar el aprendizaje dentro de una actividad interpersonal y social, donde la relación alumno-alumno se dé en un entorno comunicativo y funcional.

A partir del lenguaje la comunicación entre iguales, ofrece condiciones para que los alumnos con NEE aprendan a utilizarlo como un instrumento de aprendizaje, el cual se sirve, de valores como el respeto y la igualdad (Rogers, 1991).

Galloway (1992), considera que en el aula regular se deben de promover las actividades 
colaborativas, porque los alumnos con NEE se verán beneficiados al brindarles las facilidades para aprender con otros y ayudar a otros a aprender, además del reconocimiento de su esfuerzo y con ello favorecer su autoestima y empatía.

\section{Interacción alumno con NEE-grupo}

En este aspecto se puede identificar que los tres docentes promueven la interacción del alumno con NEE de manera grupal, a través de la participación que ofrezcan al grupo durante el trato de los contenidos curriculares.

Galloway (1992), argumenta que la integración tiene que ver con las oportunidades de los alumnos con NEE de interactuar socialmente con su profesor y compañeros de clase, esto se relaciona con la capacidad de establecer buenas relaciones a partir de una actitud asertiva de los involucrados, en un clima de trabajo respetuoso y motivador.

Otra forma de interacción que utiliza MC, se relaciona con la delegación de responsabilidades a J en torno a la entrega de algún material al resto del grupo, lo cual, le permite al alumno desplazarse por los lugares de sus compañeros y así reconocerlos, ya que, comentó la docente, en un principio esto se le dificultaba.

\section{La comunicación a través de la conversación}

En este aspecto se rescata el uso de la conversación por MA y MC para propiciar el aprendizaje a partir de los errores que son cometidos por los propios alumnos con NEE, los cuales, son una oportunidad para mejorar y no fueron utilizados para poner en evidencia a los estudiantes. Además, la conversación es guiada a partir de una serie de cuestionamientos que permiten a los estudiantes descubrir información, conocer, investigar y aportar conocimientos. En el caso de MB, utiliza la conversación como medio para disminuir las conductas agresivas del alumno R.

En los tres casos estudiados se valoran las aportaciones de los estudiantes con NEE, lo cual refleja el rol que está desempeñando cada docente en función de la integración educativa a partir de un estilo de comunicación asertiva, donde se privilegia el respeto por la opinión de los demás, puesto que es la expresión directa, honesta, adecuada y oportuna de las ideas, sentimientos, necesidades o derechos.

\section{La motivación a través de la expresión verbal y no verbal}

La motivación se identificó a partir del uso de expresiones verbales y no verbales en los tres casos. La expresión verbal y no verbal la realizan de manera simultánea y son utilizadas como un reforzamiento de su conducta o de la realización de un trabajo, donde reconocen el esfuerzo y dedicación puesta en él; lo cual, favorece el aprendizaje y una conducta productiva.

\section{B. Proceso de enseñanza-aprendizaje}

En esta categoría se describe el proceso de enseñanza-aprendizaje a partir de dos temas centrales que son: las adecuaciones curriculares y el desempeño profesional. Las adecuaciones curriculares se analizan a partir del trabajo en el aula y el extraescolar, la instrumentación didáctica donde se planifican las situaciones de aprendizaje y la evaluación. El desempeño profesional se describe a partir de las actitudes de los docentes en la integra- 
ción educativa y sobre la capacitación para fortalecer sus competencias profesionales.

La información de cada una de las subcategorías se retoma de los datos obtenidos en la bitácora de observación y en las entrevistas interactivas no estructuradas aplicadas a los tres educadores.

\section{Adecuaciones curriculares en el trabajo áulico}

En esta subcategoría se distingue que los tres casos realizan una ayuda directa en el cuaderno de trabajo de los alumnos con NEE, anotando el ejercicio que se está realizando, además de llevar a cabo algunas orientaciones verbales. Brennan (1988), considera que los alumnos con NEE pueden seguir el currículum oficial siempre y cuando se les brinde un apoyo adecuado que va más allá del registro escrito de un ejercicio, es decir, se requiere promover modificaciones en torno al tratamiento del currículum.

En el caso de MB se encontró que al alumno $\mathrm{P}$, le aplica ejercicios diferentes al resto del grupo, los cuales han sido adaptados por $\mathrm{MB}$ para trabajar los contenidos de cada bloque. Esta propuesta curricular adaptada le permite tener un mejor manejo de los procesos de enseñanza y aprendizaje, donde las posibilidades de obtener éxito en su trabajo son aún mayores, ya que se consideran sus áreas de oportunidad y de mejoramiento.

Por otra parte, MC trabaja de manera conjunta y coordinada con la mamá de J, ya que se observó su presencia y participación dentro de las actividades propuestas en el aula, lo cual, le permite a J tener un apoyo más individualizado y orientado en el trabajo propuesto por MC, pero sin demeritar el papel que tiene en el proceso de enseñanza-aprendizaje, sino para favorecerlo a partir de la ayuda que le otorga MF.

\section{Adecuaciones curriculares en el trabajo extraescolar}

Del caso de MC fue el único que se obtuvo información, a partir de la entrevista interactiva no estructurada, sobre las adecuaciones en el trabajo extraescolar, donde al alumno J se le asignan tareas diferentes a las del resto de los estudiantes de su clase, las cuales van ligadas con el contenido educativo, lo que varía es el grado de dificultad con el que se manejan, ya que es diferente por sus capacidades y características cognitivas.

\section{Adecuaciones curriculares en la instrumentación didáctica}

A partir de las entrevista se rescata que las adecuaciones curriculares que elaboran para trabajar son nulas en el caso de MA, donde comentó: "En mi planeación no escribo adecuaciones, esas las hago cuando realizo el trabajo con los alumnos aquí en el salón". En el caso de $\mathrm{MB}$ y $\mathrm{MC}$, las realizan enfocándose al manejo de situaciones de aprendizaje, donde MB dijo: "No las pongo de una manera puntual, sólo en el espacio de observaciones ahí anoto algunas adaptaciones que hago en la aplicación de alguna actividad o ejercicio". En el caso de MC mencionó: "tengo que realizar algunas adecuaciones ya sea dentro de la planeación o en los ejercicios que les pongo".

Barabtarlo y Zedansky (1995) consideran que la instrumentación didáctica ha de ser susceptible a modificaciones para el desarrollo de las estructuras cognitivas de los estudiantes, por tanto es una tarea del docente 
el constante replanteamiento de situaciones didácticas adecuadas a las necesidades de los estudiantes.

Existen en los tres casos coincidencias en el sentido que opinan que las adecuaciones curriculares las deben de realizar en conjunto con el docente de educación especial, Wenger maneja que "una práctica compartida conecta a los participantes de maneras diversas y complejas" (2001: 104). Por lo tanto, la interacción que se establezca entre ambos docentes será una pieza clave para enfrentar los retos y las oportunidades de mejorar la práctica educativa y con ello beneficiar a los usuarios con NEE.

El desarrollar una práctica compartida requiere de un compromiso mutuo y la negociación en el desarrollo de un plan de trabajo por ambas partes (Wenger, 2001), trabajar bajo un enfoque colaborativo, coordinado y sustentable para lograr la integración educativa y social de una manera eficaz.

En este sentido, si ambas partes, le proporcionan y le facilitan al alumno con NEE, las condiciones físicas, cognitivas, afectivas y sociales, se favorecerá su proceso educativo y sus necesidades serán cubiertas con acciones adecuadas y situadas a su condición como estudiante y como persona única.

\section{Adecuaciones curriculares en la evaluación}

En este rubro se destaca que en los tres casos estudiados consideran que los criterios de evaluación usados para valorar a los alumnos con NEE son diferentes porque el proceso se enfoca a un grado de logro diferente que al resto del grupo.
La aplicación del instrumento auxiliar en la evaluación del bimestre septiembre-octubre estuvo a cargo de las maestras de UsAER, las cuales utilizaron el formato estándar asignado por la mesa técnica de esta entidad, lo que modificaron fue la presentación de los puntos a valorar. Donde se destaca la importancia de iniciar con la elaboración de un examen acorde a las capacidades, condiciones y procesos de los estudiantes con NEE. Los docentes responsables mostraron inconformidad en relación con este suceso, ya que ellos consideran que es necesario realizar adecuaciones que trasciendan la aplicación guiada de la prueba. "Algunas [...] adecuaciones pueden ser relativamente superficiales, por lo que no varía demasiado la planificación o programación establecida por los maestros para todo el grupo; otras, en cambio, pueden ser sustanciales, dependiendo de las necesidades educativas especiales de los alumnos, lo cual implica una individualización del currículo" (García y Escalante, 2000: 138).

\section{Desempeño profesional a partir de la capacitación}

En el caso de MA percibe a la capacitación como una actividad personal y profesional porque le permite mejorar sus prácticas de enseñanza a partir de la búsqueda de diferentes fuentes de información. Por su parte, MB la considera como una forma de desarrollar nuevas capacidades, conocimientos, habilidades y actitudes para el trabajo docente. En lo que respecta a $\mathrm{MC}$, la maneja como una relación del binomio teoría-práctica, porque tiene que ser fundamentada y funcional para responder las necesidades educativas de los estudiantes. 
En los tres casos existe una coincidencia al exponer a la capacitación como una actividad permanente, a través de cursos, talleres o reuniones de consejo técnico pedagógico. Perrenoud (2004) establece que la formación del profesorado es un proceso que dura toda la vida, sin embargo, cada maestro vive esta premisa de una manera distinta.

\section{Servicios de Educación Especial}

En esta unidad de análisis se hace referencia a los servicios de apoyo que se prestan en la institución educativa, donde la Unidades de Servicios de Apoyo a la Educación Regular (UsAer), se enfoca al proceso de integración educativa de las alumnas y alumnos que presentan necesidades educativas especiales. En este sentido se destaca el servicio de los profesores de apoyo de forma externa al aula regular, identificado a partir del registro de observación y con los datos de las entrevistas realizadas.

\section{Servicio de apoyo externo al aula}

El apoyo externo que se les brinda a los alumnos con NEE muchas veces no es planificado de forma conjunta con el maestro del aula regular. En el caso de MC hay una negación a que salga el alumno fuera del aula, lo cual justifica a partir de un trabajo colaborativo desarrollado con la madre de familia. En los casos de MA y MB la información que se obtuvo fue a partir de la entrevista, donde manifestaron que los alumnos salían del aula para trabajar con las docentes de USAER.

Gortázar (1995) señala que el profesor de apoyo debe realizar su función en tres niveles: en el centro, con el maestro y con el alumno; además de establecer distintas refuerzos pedagógicos: previo al tratamiento de contenidos; simultáneo dentro del aula; posterior al tratamiento de contenidos; previo y posterior a la explicación de contenidos.

En este estudio se visualiza en cada uno de los casos que el servicio de apoyo que ofrece el equipo de UsAer presenta una desarticulación entre el docente del aula regular y el profesor de apoyo, ya que no existe para los docentes regulares un claro conocimiento de las funciones del equipo de apoyo, de la integración, de un plan de trabajo coordinado y del establecimiento de acuerdos para colaborar.

En todos los casos expresaron que la responsabilidad del proceso de enseñanzaaprendizaje recae en ellos, ya que son los encargados del diseño, aplicación y evaluación de estrategias didácticas.

En este sentido, el maestro del aula regular y el profesor de apoyo no conforman una comunidad de práctica activa, negociada y comprometida para lograr un objetivo común: la integración de alumnos con NEE. Wenger maneja que "una práctica compartida conecta a los participantes de maneras diversas y complejas" (2001: 104), por lo tanto, el desarrollar una práctica con estas características requiere de un compromiso mutuo y la negociación en el desarrollo de un plan de trabajo por ambas partes; trabajar bajo un enfoque colaborativo, coordinado y sustentable para lograr la integración educativa y social de una manera eficaz.

\section{Conclusiones y recomendaciones}

Los resultados muestran que el proceso de integración educativa se va construyendo en la vida cotidiana de la escuela y es definido 
a partir de las situaciones psicopedagógicas que en ella se suscitan, con la articulación que existe entre interacción, comunicación y motivación, así como también, un proceso de enseñanza-aprendizaje estratégico y el apoyo de los Servicios de Educación Especial, los cuales, forman la parte fundamental en la inclusión social de los alumnos con NEE.

Se insiste entonces en considerar al aula, un lugar de aprendizaje multidireccional que ha de compartir una gran diversidad de objetivos y tareas de aprendizaje a adquirir dentro de un entorno social diverso tanto por el origen y capacidades de los alumnos como por sus intereses y expectativas, así como la capacidad para comunicárselos entre sí. Es relevante mencionar que en cualquier tarea, los alumnos motivados intrínsecamente suelen: hacer la tarea por iniciativa propia sin esperar refuerzos positivos o premios, dedicarle tiempo a la actividad a fin de aprender más significativamente y no de memoria, ser creativos en la realización de tareas o en las participaciones que hacen en clase, persistir a pesar del fracaso (Ormrod, 2005).

En el proceso de enseñanza-aprendizaje dentro de un aula integradora se identifica que para atender a los alumnos con NEE, es necesario considerar una propuesta curricular adaptada, que se enfoca a reorganizar o modificar contenidos para hacerlos más accesibles a los estudiantes en función de sus características y de los apoyos y/o recursos didácticos disponibles.

En cuanto al rol como docentes, la integración de alumnos con NEE exige tener una actitud de apertura y de disposición favorable hacia la atención de alumnos con NEE, seguirse preparando, tener disposición de trabajar colaborativamente con compañeros docentes, padres de familia y los integrantes de UsAer.

Finalmente, muchas veces se confunde el concepto de NEE, aplicándolo únicamente a la discapacidad, por lo que muchos docentes creen que atender a los alumnos con NEE se limita a la identificación en aislado de sus discapacidades y de las causas que las determinan, imposibilitando de esa forma la generación de una actitud de aceptación y de flexibilidad hacia los alumnos que presentan esta condición. En cambio, si además de las causas, se consideran las potencialidades y limitaciones de dichos estudiantes, y se sensibiliza al resto de la comunidad escolar sobre la importancia de la integración, se estará favoreciendo la convivencia en un clima de aceptación y respeto hacia estos alumnos.

Los profesores, alumnos y padres de familia requieren un trabajo constante y en conjunto dentro de las aulas escolares, convirtiéndose en una de las formas de cambiar esta realidad para las generaciones presentes $\mathrm{y}$ futuras.

\section{Bibliografía}

Barabtarlo, A., y Zedansky (1995). Investigación y acción: una nueva didáctica para la formación de profesores. México: UNAM.

Brennan, J. (1988). El curriculum para niños con necesidades especiales. Madrid: Siglo XXI.

Bruner, J. (1984). Los formatos de la adquisición del lenguaje. En Acción, pensamiento y lenguaje. Barcelona: Alianza.

Galloway, D. (1992). La interacción con los niños con necesidades educativas especiales. Psicología social de la escuela primaria. 3a Ed., 231-246. (C. Rogers, y P. Kutnick, copiladores) Barcelona: Paidós.

García, C. I., y Escalante, H. I. (2000). La integración educativa en el aula. Principios, finalidades y estrategias. México: SEP, Fondo Mixto de Cooperación Técnica y Científica MéxicoEspaña.

Gortázar, A. (1995). El profesor de apoyo en la escuela ordinaria. Desarrollo psicológico y educación, III. Necesidades educativas especiales y aprendizaje escolar, 8 ${ }^{\mathrm{a}}$ Ed, 367-382. (A. Mar- 
REVISTA DE INVESTIGACIÓN EDUCATIVA DE LA REDIECH N. 4

chesi, C. Coll, y J. Palacios, copiladores) Madrid: Alianza.

Hernández, S. R. (1998). Manual de investigación social. México: Trillas.

Hernández, S. R., Fernández, C. C., y Baptista, L. P. (2006). Metodología de investigación. México: McGraw-Hill.

Marchesi, A., y Martin, E. (1995). Del lenguaje del trastorno a las necesidades educativas especiales. Desarrollo Psicológico y Educación, III. Necesidades Educativas Especiales y Aprendizaje Escolar, 8a. Ed., 15-33. (Á. Marchesi, C. Coll, y J. Palacios, copiladores) Madrid, Alianza.

Mayan, M. J. (2001). Una introducción a los métodos cualitativos: un módulo de entrenamiento para estudiantes y profesionales. Recuperado el 1 de Septiembre de 2010, de Universidad de Alberta: http://www.ualberta.ca/ iiqm//pdfs/introduccion.pdf

Ormrod, J. E. (2005). Aprendizaje Humano. 4a Ed., Madrid: Pearson Educación.

Perrenoud, P. (2004). Diez nuevas competencias para enseñar. Méxi- co, SEP.

Pourtois, J. y Huguette D. (1992). La legitimación de los conocimientos. Epistemología e instrumentación en ciencias humanas. Barcelona, Herder.

Rogers, C. R. (1991). Las relaciones interpersonales en la facilitación del aprendizaje. En Libertad y creatividad de la educación en la década de los ochenta. Barcelona: Paidós.

Saint-Onge, M. (2000). Yo explico, pero ellos...¿aprenden? México, SEP, Biblioteca para la Actualización del Maestro.

SEP (2006). Orientaciones generales para el funcionamiento de los servicios de educación especial. México: SEP, Programa Nacional de Fortalecimiento de la Educación Especial y de la Integración Educativa.

SEP (2002). Programa Nacional de Fortalecimiento de la Educación Especial y de la Integración Educativa. México.

Wenger, E. (2001). Comunidades de práctica, aprendizaje, significado e identidad. En Cognición y desarrollo humano. España: Paidós. 